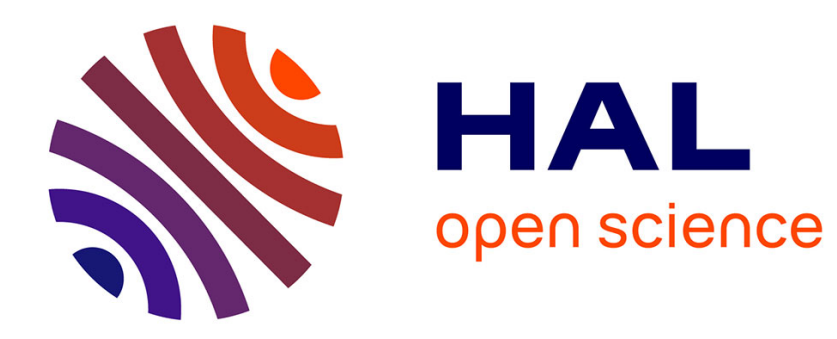

\title{
Prescribed Szlenk index of separable Banach spaces
} R M Causey, Gilles Lancien

\section{To cite this version:}

R M Causey, Gilles Lancien. Prescribed Szlenk index of separable Banach spaces. Studia Mathematica, 2019, 248 (2), pp.108-127. 10.4064/sm171012-9-9 . hal-01753837

\section{HAL Id: hal-01753837 https://hal.science/hal-01753837}

Submitted on 29 Mar 2018

HAL is a multi-disciplinary open access archive for the deposit and dissemination of scientific research documents, whether they are published or not. The documents may come from teaching and research institutions in France or abroad, or from public or private research centers.
L'archive ouverte pluridisciplinaire HAL, est destinée au dépôt et à la diffusion de documents scientifiques de niveau recherche, publiés ou non, émanant des établissements d'enseignement et de recherche français ou étrangers, des laboratoires publics ou privés. 


\title{
PRESCRIBED SZLENK INDEX OF ITERATED DUALS
}

\author{
R.M. CAUSEY AND G. LANCIEN
}

\begin{abstract}
In a previous work, the first named author described the set $\mathcal{P}$ of all values of the Szlenk indices of separable Banach spaces. We complete this result by showing that for any integer $n$ and any ordinal $\alpha$ in $\mathcal{P}$, there exists a separable Banach space $X$ such that the Szlenk of the dual of order $k$ of $X$ is equal to the first infinite ordinal $\omega$ for all $k$ in $\{0, . ., n-1\}$ and equal to $\alpha$ for $k=n$. One of the ingredients is to show that the Lindenstrauss space and its dual both have a Szlenk index equal to $\omega$.
\end{abstract}

\section{INTRODUCTION AND NOTATION}

In this paper we exhibit some new properties of the Szlenk index, an ordinal index associated with a Banach space. More precisely we study the values that can be achieved as a Szlenk index of a Banach space and of its iterated duals. Let us first recall the definition of the Szlenk index.

Let $X$ be a Banach space, $K$ a weak*-compact subset of its dual $X^{*}$ and $\varepsilon>0$. Then we define

$$
s_{\varepsilon}^{\prime}(K)=\left\{x^{*} \in K, \text { for any weak }{ }^{*} \text { neighborhood } U \text { of } x^{*}, \operatorname{diam}(K \cap U) \geq \varepsilon\right\}
$$

and inductively the sets $s_{\varepsilon}^{\alpha}(K)$ for $\alpha$ ordinal as follows: $s_{\varepsilon}^{\alpha+1}(K)=s_{\varepsilon}^{\prime}\left(s_{\varepsilon}^{\alpha}(K)\right)$ and $s_{\varepsilon}^{\alpha}(K)=\bigcap_{\beta<\alpha} s_{\varepsilon}^{\beta}(K)$ if $\alpha$ is a limit ordinal.

Then $S z(K, \varepsilon)=\inf \left\{\alpha, s_{\varepsilon}^{\alpha}(K)=\emptyset\right\}$ if it exists and we denote $S z(K, \varepsilon)=\infty$ otherwise. Next we define $S z(K)=\sup _{\varepsilon>0} S z(K, \varepsilon)$. The closed unit ball of $X^{*}$ is denoted $B_{X^{*}}$ and the Szlenk index of $X$ is $S z(X)=S z\left(B_{X^{*}}\right)$.

The Szlenk index was first introduced by W. Szlenk [19], in a slightly different form, in order to prove that there is no separable reflexive Banach space universal for the class of all separable reflexive Banach spaces. The key ingredients in [19] are that the Szlenk index of a separable reflexive space is always countable and that for any countable ordinal $\alpha$, there exists a separable reflexive Banach space with Szlenk index larger than $\alpha$. It has been remarked in [13] that, when it is different from $\infty$, the Szlenk index of a Banach space is always of the form $\omega^{\alpha}$, for some ordinal $\alpha$. Here, $\omega$ denotes the first infinite ordinal. On the other hand, it follows from the work of Bessaga and Pełczyński [2] and Samuel [18] that if $K$ is an infinite, countable, compact topological space, then the Szlenk index of the space of continuous functions on $K$ is $\omega^{\alpha+1}$, where $\alpha$ is the unique countable ordinal such that $\omega^{\alpha} \leq C B(K)<\omega^{\alpha+1}$ and

2010 Mathematics Subject Classification. 46B20.

The second named author was supported by the French "Investissements d'Avenir" program, project ISITE-BFC, contract ANR-15-IDEX-03. 
$C B(K)$ is the Cantor-Bendixson index of $K$. Finally, the set of all possible values for the Szlenk index of a Banach space was completely described in [5] (Theorem 1.5). One consequence of this general result is that for any countable ordinal $\alpha$, there exists an infinite dimensional separable Banach space $X$ with $S z(X)=\alpha$ if and only if $\alpha \in \Gamma \backslash \Lambda$, where

$$
\Gamma=\left\{\omega^{\xi}, \xi \in\left[1, \omega_{1}\right)\right\} \text { and } \Lambda=\left\{\omega^{\omega^{\xi}}, \xi \in\left[1, \omega_{1}\right) \text { and } \xi \text { is a limit ordinal }\right\} .
$$

Our main result shows that there is quite some freedom in prescribing the Szlenk indices of the iterated duals of a separable Banach space. We shall use the notation $Z^{(n)}$ for the $n^{\text {th }}$ dual of a Banach space $Z$. Then our statement is the following.

Theorem 1.1. Let $n \in \mathbb{N}$ and $\alpha \in \Gamma \backslash \Lambda$. Then there exists a separable Banach space $Z$ such that for all $k \in\{0, . ., n-1\}, S z\left(Z^{(k)}\right)=\omega$ and $S z\left(Z^{(n)}\right)=\alpha$.

This result relies on two statements that have their own interest.

We recall that J. Lindenstrauss proved in [14] that for any separable Banach space $X$, there exists a Banach space $Z$ such that $Z^{* *} / Z$ is isomorphic to $X$. Our first step is to study the Szlenk index of this space and of its dual. More precisely, in section 2, we prove:

Theorem 1.2. For any separable Banach space $X$, the associated Lindenstrauss space $Z$ satisfies the following property: $S z(Z)=S z\left(Z^{*}\right)=\omega$.

Our second step is the following refinement of Theorem 1.5 from [5] that we prove in subsection 3.1.

Theorem 1.3. For any $\alpha \in \Gamma \backslash \Lambda$ there exists a separable Banach space $G_{\alpha}$ such that $S z\left(G_{\alpha}\right)=\omega$ and $S z\left(G_{\alpha}^{*}\right)=\alpha$. Moreover, $G_{\alpha}$ can be taken to be reflexive with an unconditional basis.

Then we will quickly deduce the proof of Theorem 1.1 in subsection 3.2.

We conclude this introduction by recalling the definitions of some uniform asymptotic properties of norms that we will use. For a Banach space $(X,\|\|)$ we denote by $B_{X}$ the closed unit ball of $X$ and by $S_{X}$ its unit sphere. The following definitions are due to V. Milman [16] and we follow the notation from [11]. For $t \in[0, \infty), x \in S_{X}$ and $Y$ a closed linear subspace of $X$, we define

$$
\bar{\rho}_{X}(t, x, Y)=\sup _{y \in S_{Y}}(\|x+t y\|-1) \quad \text { and } \quad \bar{\delta}_{X}(t, x, Y)=\inf _{y \in S_{Y}}(\|x+t y\|-1) .
$$

Then

$$
\bar{\rho}_{X}(t, x)=\inf _{\operatorname{dim}(X / Y)<\infty} \bar{\rho}_{X}(t, x, Y) \quad \text { and } \quad \bar{\delta}_{X}(t, x)=\sup _{\operatorname{dim}(X / Y)<\infty} \bar{\delta}_{X}(t, x, Y)
$$

and

$$
\bar{\rho}_{X}(t)=\sup _{x \in S_{X}} \bar{\rho}_{X}(t, x) \quad \text { and } \quad \bar{\delta}_{X}(t)=\inf _{x \in S_{X}} \bar{\delta}_{X}(t, x)
$$

The norm \|\| is said to be asymptotically uniformly smooth (in short AUS) if

$$
\lim _{t \rightarrow 0} \frac{\bar{\rho}_{X}(t)}{t}=0 .
$$


It is said to be asymptotically uniformly convex (in short AUC) if

$$
\forall t>0 \quad \bar{\delta}_{X}(t)>0 .
$$

Let $p \in(1, \infty)$ and $q \in[1, \infty)$.

We say that the norm of $X$ is $p$-AUS if there exists $c>0$ such that for all $t \in[0, \infty)$, $\bar{\rho}_{X}(t) \leq c t^{p}$.

We say that the norm of $X$ is $q$-AUC if there exits $c>0$ such that for all $t \in[0,1]$, $\bar{\delta}_{X}(t) \geq c t^{q}$.

Similarly, there is on $X^{*}$ a modulus of weak* asymptotic uniform convexity defined by

$$
\bar{\delta}_{X}^{*}(t)=\inf _{x^{*} \in S_{X^{*}}} \sup _{E} \inf _{y^{*} \in S_{E}}\left(\left\|x^{*}+t y^{*}\right\|-1\right),
$$

where $E$ runs through all weak ${ }^{*}$-closed subspaces of $X^{*}$ of finite codimension. The norm of $X^{*}$ is said to be weak ${ }^{*}$ uniformly asymptotically convex (in short weak ${ }^{*}$-AUC) if $\bar{\delta}_{X}^{*}(t)>0$ for all $t$ in $(0, \infty)$. If there exists $c>0$ and $q \in[1, \infty)$ such that for all $t \in[0,1] \bar{\delta}_{X}^{*}(t) \geq c t^{q}$, we say that the norm of $X^{*}$ is $q$-weak ${ }^{*}$-AUC.

Let us recall the following classical duality result concerning these moduli (see for instance [8] Corollary 2.3 for a precise statement).

Proposition 1.4. Let $X$ be a Banach space.

Then \|\|$_{X}$ is AUS if and and only if \|\|$_{X^{*}}$ is weak ${ }^{*}-A U C$.

If $p, q \in(1, \infty)$ are conjugate exponents, then \|\|$_{X}$ is $p$-AUS if and and only if \|\|$_{X^{*}}$ is $q$-weak ${ }^{*}-A U C$.

Finally let us recall the following fundamental result, due to Knaust, Odell and Schlumprecht [12], which relates the existence of equivalent asymptotically uniformly smooth norms and the Szlenk index.

Theorem 1.5 (Knaust-Odell-Schlumprecht).

Let $X$ be a separable infinite dimensional Banach space. Then $X$ admits an equivalent norm which is asymptotically uniformly smooth if and only if $S z(X)=\omega$.

\section{The Szlenk index And the Lindenstrauss SPACE}

We recall the construction given by J. Lindenstrauss in [14] (see also [15] Theorem 1.d.3).

Let $\left(X,\|\|_{X}\right)$ be a separable Banach space and fix $\left(x_{i}\right)_{i=1}^{\infty}$ a dense sequence in the unit sphere $S_{X}$ of $X$. Let $E$ be defined by

$$
E=\left\{a=\left(a_{i}\right)_{i=1}^{\infty} \in \mathbb{R}^{\mathbb{N}}, \quad\|a\|_{E}=\sup _{0=p_{0}<p_{1}<. .<p_{k}}\left(\sum_{j=1}^{k}\left\|\sum_{i=p_{j-1}+1}^{p_{j}} a_{i} x_{i}\right\|_{X}^{2}\right)^{1 / 2}<\infty\right\} .
$$

Then $\left(E,\|\|_{E}\right)$ is a Banach space. Let us denote $\left(e_{i}\right)_{i=1}^{\infty}$ the canonical algebraic basis of $c_{00}$, the space of finitely supported real valued sequences. It is clear that $\left(e_{i}\right)_{i=1}^{\infty}$ is a boundedly complete basis of $E$. It follows that $E$ is isometric to the dual $Y^{*}$ of a Banach space $Y$ with a shrinking basis. If $\left(e_{i}^{*}\right)_{i=1}^{\infty}$ is the sequence of coordinate functionals associated with the basis $\left(e_{i}\right)_{i=1}^{\infty}$ of $E$, then the canonical image of $Y$ in its bidual $Y^{* *}$ is the closed linear span of $\left\{e_{i}^{*}, i \geq 1\right\}$ and $\left(e_{i}^{*}\right)_{i=1}^{\infty}$ can be seen as the 
shrinking basis of $Y$.

Note now that if $a=\left(a_{i}\right)_{i=1}^{\infty} \in E$, then the series $\sum_{i=1}^{\infty} a_{i} x_{i}$ is converging in $X$. It then follows from the density of $\left(x_{i}\right)_{i=1}^{\infty}$ in $S_{X}$ that the map $Q: E \rightarrow X$, defined by $Q(a)=\sum_{i=1}^{\infty} a_{i} x_{i}$ is linear, onto and satisfies $\|Q\|=1$. Therefore $Q^{*}$ is an isometry from $X^{*}$ into $Y^{* *}$. The main result of [14] is that $Y^{* *}=Y \oplus Q^{*}\left(X^{*}\right)$, where $Y$ is the canonical image of $Y$ in $Y^{* *}$, and the projection from $Y^{* *}$ onto $Q^{*}\left(X^{*}\right)$ with kernel $Y$ has norm one. In particular, $Y$ is isomorphic to the quotient space $Y^{* *} / Q^{*}\left(X^{*}\right)$.

Now let $Z$ denote the kernel of $Q$. The space $Z$ is a subspace of $E=Y^{*}$ and its orthogonal $Z^{\perp}$ is clearly equal to $Q^{*}\left(X^{*}\right)$. It follows from the classical duality theory that $Z^{*}$ is isometric to $Y^{* *} / Q^{*}\left(X^{*}\right)$ and therefore isomorphic to $Y$. If $I$ is the inclusion map from $Z$ into $Y^{*}$ and $J_{Y}$ is the canonical injection from $Y$ into $Y^{* *}$, an isomorphism from $Y$ onto $Z^{*}$ is given by $T=I^{*} J_{Y}$. Finally, if $J_{Z}$ is the canonical injection from $Z$ into $Z^{* *}$, it is easy to check that $T^{*} J_{Z}=I$. It follows immediately that $Z^{* *} / J_{Z}(Z)$ (or simply $\left.Z^{* *} / Z\right)$ is isomorphic to $Y^{*} / Z$ and therefore to $X$.

The purpose of this section is to prove Theorem 1.2. In fact, our result is more precise.

Theorem 2.1. For any separable Banach space $X$, the associated Lindenstrauss space $Z$ satisfies the following properties.

(i) The space $Z^{*}$ admits an equivalent norm which is 2-AUS.

(ii) The space $Z$ admits an equivalent norm which is 2-AUS.

We start with the proof of the easy part (i) which can be precisely stated as follows.

Proposition 2.2. The norm \|\|$_{E}$ is 2-weak ${ }^{*}-A U C$ on $Y^{*}=E$ and therefore \|\|$_{Y}$ is 2-AUS. In particular, $Z^{*}$ admits an equivalent norm which is 2-AUS, there exists $C>0$ such that for all $\varepsilon>0, S z\left(Z^{*}, \varepsilon\right) \leq C \varepsilon^{-2}$, and $S z(Y)=S z\left(Z^{*}\right)=\omega$.

This result is an immediate consequence of the following elementary lemma.

Lemma 2.3. Let $a, b \in E$ and assume that there exits $k \in \mathbb{N}$ such that the sequence $a$ is supported in $[1, k]$ while the sequence $b$ is supported in $[k+3, \infty)$. Then

$$
\|a+b\|_{E}^{2} \geq\|a\|_{E}^{2}+\|b\|_{E}^{2}
$$

Proof. Since $a$ is supported in $[1, k]$ we can find a sequence $0=p_{0}<p_{1}<. .<p_{m}=k+1$ such that

$$
\|a\|_{E}^{2}=\sum_{j=1}^{k}\left\|\sum_{i=p_{j-1}+1}^{p_{j}} a_{i} x_{i}\right\|_{X}^{2}
$$

Fix $\eta>0$. Since $b$ is supported in $[k+3, \infty)$ we can find a sequence $k+1=q_{0}<q_{1}<$ ..$<q_{r}$ such that

$$
\|b\|_{E}^{2} \geq \sum_{j=1}^{r}\left\|\sum_{i=q_{j-1}+1}^{q_{j}} b_{i} x_{i}\right\|_{X}^{2}-\eta
$$

Denote $n_{0}=0, n_{j}=p_{j}$ for $j \leq m$ and $n_{j}=q_{j-m}$ for $m \leq j \leq m+r$. Then

$$
\|a+b\|_{E}^{2} \geq \sum_{j=1}^{m+r}\left\|\sum_{i=n_{j-1}+1}^{n_{j}}(a+b)_{i} x_{i}\right\|_{X}^{2} \geq\|a\|_{E}^{2}+\|b\|_{E}^{2}-\eta .
$$


This finishes the proof.

Remark. The above statement can be rephrased as follows. The space $Z^{*}$ admits an equivalent norm whose dual norm is 2 -weak ${ }^{*}$-AUC. It is important to note that this norm cannot be a dual norm of an equivalent norm on $Z$. Indeed a bidual norm cannot be $\mathrm{AUC}^{*}$ unless the space is reflexive, which means in our case that $X=\{0\}$. In particular, in Lindenstrauss' construction, the space $Y$ is isomorphic but never isometric to $Z^{*}$.

We now turn to the proof of part (ii) in Theorem 2.1. The proof will first rely on the following technical lemma.

Lemma 2.4. Let $a^{1}, . ., a^{N}$ in $E$ and $\varepsilon_{1}, . ., \varepsilon_{N}>0$, where $a^{k}=\left(a_{i}^{k}\right)_{i=1}^{\infty}$, for $1 \leq k \leq N$. Assume that there exist $0=r_{0}<r_{1}<. .<r_{N}$ so that

$$
\forall k \in\{1, . ., N\}, \operatorname{supp}\left(a^{k}\right) \subset\left(r_{k-1}, r_{k}\right) \text { and }\left\|\sum_{i \in \operatorname{supp}\left(a^{k}\right)} a_{i}^{k} x_{i}\right\|_{X} \leq \varepsilon_{k} .
$$

Then

$$
\left\|\sum_{k=1}^{N} a^{k}\right\|_{E} \leq \sum_{k=1}^{N} \varepsilon_{k}+2\left(\sum_{k=1}^{N}\left\|a^{k}\right\|_{E}^{2}\right)^{1 / 2}
$$

Proof. Fix $0=p_{0}<. .<p_{m}$ and assume without lost of generality that $p_{m} \geq r_{N}$. Then for $j \in\{1, . ., m\}$ we denote

$$
A_{j}=\left\{k \leq N,\left(r_{k-1}, r_{k}\right) \subset\left(p_{j-1}, p_{j}\right]\right\}, \quad A=\bigcup_{j=1}^{m} A_{j} \text { and } B=\{1, \cdots, m\} \backslash A .
$$

We first estimate

$$
\begin{gathered}
\left(\sum_{j=1}^{m}\left\|\sum_{i=p_{j-1}+1}^{p_{j}}\left(\sum_{k \in A} a_{i}^{k}\right) x_{i}\right\|_{X}^{2}\right)^{1 / 2} \leq \sum_{j=1}^{m}\left\|\sum_{i=p_{j-1}+1}^{p_{j}}\left(\sum_{k \in A} a_{i}^{k}\right) x_{i}\right\|_{X} \\
=\sum_{j=1}^{m}\left\|\sum_{k \in A_{j}} \sum_{i=p_{j-1}+1}^{p_{j}} a_{i}^{k} x_{i}\right\|_{X} \leq \sum_{j=1}^{m} \sum_{k \in A_{j}}\left\|a^{k}\right\|_{X} \leq \sum_{k=1}^{N}\left\|a^{k}\right\|_{X} .
\end{gathered}
$$

We have proved that

$$
\left(\sum_{j=1}^{m}\left\|\sum_{i=p_{j-1}+1}^{p_{j}}\left(\sum_{k \in A} a_{i}^{k}\right) x_{i}\right\|_{X}^{2}\right)^{1 / 2} \leq \sum_{k=1}^{N} \varepsilon_{k} .
$$

We now relabel $\left(a^{k}\right)_{k \in B}$ as $b^{1}, . ., b^{L}$ in such a way that there exists $0=s_{0}<s_{1}<. .<s_{L}$ such that for all $l \in\{1, . ., L\}, \operatorname{supp}\left(b^{l}\right) \subset\left(s_{l-1}, s_{l}\right)$ and $\left(s_{l-1}, s_{l}\right)$ is not included in any of the sets $\left(p_{j-1}, p_{j}\right]$, for $1 \leq j \leq m$. Then we construct $i_{0}<i_{1}<. .<i_{L}$ as follows.

First we set $i_{0}=s_{0}=p_{0}=0$. Then the properties of the sequences $\left(s_{l}\right)$ and $\left(p_{j}\right)$ imply 
that there exists $i_{1}>2$ such that $p_{i_{1}-1}<s_{1} \leq p_{i_{1}}$ and inductively we find, for all $l \in\{1, . ., L\}, i_{l}>i_{l-1}$ such that $p_{i_{l}-1}<s_{l} \leq p_{i_{l}}$. We can now write

$$
\sum_{j=1}^{m}\left\|\sum_{i=p_{j-1}+1}^{p_{j}}\left(\sum_{k \in B} a_{i}^{k}\right) x_{i}\right\|_{X}^{2}=\sum_{q=1}^{L} \sum_{j=i_{q-1}+1}^{i_{q}}\left\|\sum_{i=p_{j-1}+1}^{p_{j}}\left(\sum_{l=1}^{L} b_{i}^{l}\right) x_{i}\right\|_{X}^{2} .
$$

Using the convention $b^{0}=0=b^{L+1}$ and the properties of our various sequences we get

$$
\begin{gathered}
\sum_{j=1}^{m}\left\|\sum_{i=p_{j-1}+1}^{p_{j}}\left(\sum_{k \in B} a_{i}^{k}\right) x_{i}\right\|_{X}^{2}=\sum_{q=1}^{L} \sum_{j=i_{q-1}+1}^{i_{q}}\left\|\sum_{i=p_{j-1}+1}^{p_{j}}\left(b_{i}^{q}+b_{i}^{q+1}\right) x_{i}\right\|_{X}^{2} \\
\leq \sum_{q=1}^{L}\left\|b^{q}+b^{q+1}\right\|_{E}^{2} \leq 4 \sum_{q=1}^{L}\left\|b^{q}\right\|_{E}^{2} \leq 4 \sum_{k=1}^{N}\left\|a_{k}\right\|_{E}^{2},
\end{gathered}
$$

which yields

$$
\left(\sum_{j=1}^{m}\left\|\sum_{i=p_{j-1}+1}^{p_{j}}\left(\sum_{k \in B} a_{i}^{k}\right) x_{i}\right\|_{X}^{2}\right)^{1 / 2} \leq 2\left(\sum_{k=1}^{N}\left\|a_{k}\right\|_{E}^{2}\right)^{1 / 2} .
$$

The conclusion of the proof of this lemma now clearly follows from equations (2.1) and (2.2), a triangle inequality and by taking the supremum over all sequences $\left(p_{j}\right)_{j}$.

Before we proceed with the proof of Theorem 2.1, we need to introduce some notation. We denote $\mathbb{N}<\omega$ the set of finite or empty sequences in $\mathbb{N}$. For $t=\left(t_{1}, . ., t_{k}\right) \in \mathbb{N}<\omega$ and $n \in \mathbb{N},(t, n)$ denotes the sequence $\left(t_{1}, . ., t_{k}, n\right)$. Then a family $\left(x_{t}\right)_{t \in \mathbb{N}<\omega}$ in a Banach space $X$ is said to be a weakly null tree if for any $t$ in $\mathbb{N}^{<\omega}$ the sequence $\left(x_{(t, n)}\right)_{n=1}^{\infty}$ is weakly null.

Proof of (ii) in Theorem 2.1. Let $\left(z_{t}\right)_{t \in \mathbb{N}<\omega}$ be a weakly null tree in the unit ball $B_{Z}$ of $Z$. Fix $\left(\varepsilon_{n}\right)_{n=0}^{\infty}$ a sequence in $(0, \infty)$ such that $\sum_{n=0}^{\infty} \varepsilon_{n} \leq \frac{1}{4}$. By extracting a full subtree, we may assume that for any $s \in \mathbb{N}^{\mathbb{N}}$ there exist $\left(a^{s_{n}}\right)_{n=1}^{\infty}$ in $E$ and $0=r_{s_{0}}<$ $r_{s_{1}}<. .<r_{s_{n}}<.$. so that

$$
\forall k \in \mathbb{N}, \operatorname{supp}\left(a^{s_{k}}\right) \subset\left(r_{s_{k-1}}, r_{s_{k}}\right) \text { and }\left\|a^{s_{k}}-z_{s_{1}, . ., s_{k}}\right\|_{E} \leq \varepsilon_{k} .
$$

Since $\left(z_{t}\right)_{\mathbb{N}<\omega}$ is included in the kernel of $Q$, the last condition implies that

$$
\forall s \in \mathbb{N}^{\mathbb{N}} \forall k \in \mathbb{N} \quad \sum_{i \in \operatorname{supp}\left(a^{s} k\right)} a_{i}^{s_{k}} x_{i} \|_{X} \leq \varepsilon_{k}
$$

We can therefore apply Lemma 2.4 and the triangle inequality to get that for all $\left(\lambda_{t}\right)_{t \in \mathbb{N}<\omega}$ in $\mathbb{R}$ and all $s \in \mathbb{N}^{<\omega}$,

$$
\left\|\sum_{\emptyset<t \leq s} \lambda_{t} z_{t}\right\|_{E} \leq 2 \sum_{\emptyset<t \leq s}\left|\lambda_{t}\right| \varepsilon_{|t|}+2\left(\sum_{\emptyset<t \leq s} \lambda_{t}^{2}\right)^{1 / 2}
$$


It then follows from our initial choice of the sequence $\left(\varepsilon_{n}\right)_{n=0}^{\infty}$ that

$$
\forall s \in \mathbb{N}^{<\omega} \quad\left\|\sum_{\emptyset<t \leq s} \lambda_{t} z_{t}\right\|_{E} \leq 3\left(\sum_{\emptyset<t \leq s} \lambda_{t}^{2}\right)^{1 / 2} .
$$

In the terminology introduced in [7] it means that $Z$ satisfies $\ell_{2}$ upper tree estimates. It then follows from Theorem 1.1 in [7] that $Z$ admits an equivalent norm which is 2-AUS.

\section{Prescribing Szlenk indices}

\subsection{Proof of Theorem 1.3.}

We now turn to the proof of Theorem 1.3, which will take a few steps.

First we describe a general construction of a Banach space associated with a given Banach space with a Schauder basis, which will be essential in the sequel. As it will be clear, this resembles Lindenstrauss' construction.

So assume that $\left(e_{i}\right)_{i=1}^{\infty}$ is a normalized Schauder basis for the Banach space $X$ and let $X^{\ell_{2}}$ be the completion of $\operatorname{span}\left\{e_{i}: i \in \mathbb{N}\right\}$ with respect to the norm

$$
\left\|\sum_{i=1}^{\infty} a_{i} e_{i}\right\|_{X^{\ell_{2}}}=\sup \left\{\left(\sum_{i=1}^{\infty}\left\|\sum_{j=k_{i-1}+1}^{k_{i}} a_{j} e_{j}\right\|_{X}^{2}\right)^{1 / 2}: 0 \leq k_{0}<k_{1}<\ldots\right\} .
$$

This construction is presented in section 3 of [17] in a more general setting. With the notation from [17], the space $X^{\ell_{2}}$ is $Z^{V}(E)$, with $Z=X, V=\ell_{2}$ and $E$ being the finite dimensional decomposition of $X$ associated with the basis $\left(e_{i}\right)_{i=1}^{\infty}$. Clearly, the definition of $X^{\ell_{2}}$ depends on our choice of the basis $\left(e_{i}\right)_{i=1}^{\infty}$. However, we shall omit reference to this dependence in our notation.

Note first that $\left(e_{i}\right)_{i=1}^{\infty}$ is a basis for $X^{\ell_{2}}$ which is an unconditional basis for $X^{\ell_{2}}$ if $\left(e_{i}\right)_{i=1}^{\infty}$ is unconditional in $X$. Furthermore, the formal identity $I: X^{\ell_{2}} \rightarrow X$ is a well-defined operator of norm one. Note also that $\left(e_{i}\right)_{i=1}^{\infty}$ is a bimonotone basis for $X^{\ell_{2}}$, even if $\left(e_{i}\right)_{i=1}^{\infty}$ is not bimonotone in $X$.

Proposition 3.1. Assume that $\left(e_{i}\right)_{i=1}^{\infty}$ is a shrinking basis of $X$. Then

(i) $X^{\ell_{2}}$ is reflexive. In particular, $\left(e_{i}\right)_{i=1}^{\infty}$ is a shrinking and boundedly complete basis of $X^{\ell_{2}}$.

(ii) The space $\left(X^{\ell_{2}}\right)^{*}$ is 2-AUS. In particular $S z\left(\left(X^{\ell_{2}}\right)^{*}\right)=\omega$.

Proof. The statement (i) is a particular case of Corollary 3.4 in [17].

(ii) Since $\left(e_{i}\right)_{i=1}^{\infty}$ is shrinking, $\left(X^{\ell_{2}}\right)^{*}=\overline{\operatorname{span}}\left\{e_{i}^{*}: i \in \mathbb{N}\right\}$. Now it is clear that if $x^{*}, y^{*} \in\left(X^{\ell_{2}}\right)^{*}$ with $\max \operatorname{supp}\left(x^{*}\right)<\min \operatorname{supp}\left(y^{*}\right)$, then $\left\|x^{*}+y^{*}\right\|^{2} \leq\left\|x^{*}\right\|^{2}+\left\|y^{*}\right\|^{2}$. Here, the support is meant with respect to the basis $\left(e_{i}^{*}\right)_{i=1}^{\infty}$ of $\left(X^{\ell_{2}}\right)^{*}$. Hence $\left(X^{\ell_{2}}\right)^{*}$ is 2-AUS and has Szlenk index $\omega$.

Note that this also implies that the bidual norm on $\left(X^{\ell_{2}}\right)^{* *}$ is weak* AUC and reproves the fact that $X^{\ell_{2}}$ is reflexive, knowing that $\left(e_{i}\right)_{i=1}^{\infty}$ is shrinking.

Our next proposition provides a crucial estimate for $S z\left(X^{\ell_{2}}\right)$. 
Proposition 3.2. Assume that $\left(e_{i}\right)_{i=1}^{\infty}$ is a shrinking basis of $X$. Then $S z\left(X^{\ell_{2}}\right) \leq S z(X)$.

Our strategy will be to show that $S z\left(X^{\ell_{2}}\right) \leq S z\left(\ell_{2}(X)\right)$. Then the conclusion will follow from the well known fact that $S z\left(\ell_{2}(X)\right)=S z(X)$ when $X$ is infinite dimensional (see [3] for a general study of the behavior of the Szlenk index under direct sums).

Let $M_{1}$ be the set of all sequences $\left(y_{i}^{*}\right)_{i=1}^{\infty}$ in $B_{\ell_{2}\left(X^{*}\right)}$ such that there exist $n \in \mathbb{N}$ and $0=k_{0}<\cdots<k_{n-1}$ with the following properties: $y_{i}^{*} \in \operatorname{span}\left\{e_{j}^{*}, k_{i-1}<j \leq k_{i}\right\}$ for each $1 \leq i<n, y_{n}^{*}$ belongs to the closed linear span of $\left\{e_{j}^{*}, j>k_{n-1}\right\}$ and $y_{i}^{*}=0$ for all $i>n$. Then we denote by $M_{2}$ the set of all sequences $\left(y_{i}^{*}\right)_{i=1}^{\infty}$ in $B_{\ell_{2}\left(X^{*}\right)}$ such that there exits an infinite sequence $0=k_{0}<\cdots<k_{i}<\cdots$ such that for all $i \in \mathbb{N}$, $y_{i}^{*} \in \operatorname{span}\left\{e_{j}^{*}, k_{i-1}<j \leq k_{i}\right\}$. Finally, we set $M=M_{1} \cup M_{2}$.

It easy to check that $M$ is weak*-compact in $\ell_{2}\left(X^{*}\right)=\ell_{2}(X)^{*}$.

Recall that $I: X^{\ell_{2}} \rightarrow X$ denotes the formal identity and that $\|I\|=1$, and define $j: M \rightarrow\left(X^{\ell_{2}}\right)^{*}$ by

$$
\forall y^{*}=\left(y_{i}^{*}\right)_{i=1}^{\infty} \in M, \quad j\left(y^{*}\right)=\sum_{i=1}^{\infty} I^{*} y_{i}^{*}
$$

An elementary application of Cauchy-Schwarz inequality shows that $j$ is well defined and that

$$
\forall y^{*} \in M, \quad\left\|j\left(y^{*}\right)\right\|_{\left(X^{\left.\ell_{2}\right)^{*}}\right.} \leq\left\|y^{*}\right\|_{\ell_{2}\left(X^{*}\right)} .
$$

It is also easy to verify that $j$ is weak ${ }^{*}$-weak ${ }^{*}$ continuous.

So we now consider the weak ${ }^{*}$-compact subset $K=j(M)$ of $B_{\left(X^{\ell_{2}}\right)^{*}}$. We will need that $K$ is norming for $X^{\ell_{2}}$. More precisely, we have:

Claim 3.3. There exists a constant $c>0$ such that

$$
\forall x \in X^{\ell_{2}}, \quad\|x\|_{X^{\ell_{2}}} \geq c \sup _{x^{*} \in K} x^{*}(x) .
$$

Proof. Let $C \geq 1$ be the bimonotonicity constant of the Schauder basis $\left(e_{i}\right)_{i=1}^{\infty}$ of $X$, let $x=\sum_{i=1}^{\infty} a_{i} e_{i} \in X^{\ell_{2}}$ and $\varepsilon>0$. Pick $0 \leq k_{0}<\cdots<k_{n}$ such that

$$
\left(\sum_{i=1}^{n}\left\|\sum_{j=k_{i-1}+1}^{k_{i}} a_{j} e_{j}\right\|_{X}^{2}\right)^{1 / 2} \geq\|x\|_{X^{\ell_{2}}}-\varepsilon
$$

It follows from the Hahn-Banach theorem that for all $1 \leq i \leq n$, there exists $u_{i}^{*} \in X^{*}$ with $\operatorname{supp}\left(u_{i}^{*}\right) \subset\left(k_{i-1}, k_{i}\right]$ and such that

$$
u_{i}^{*}\left(\sum_{j=k_{i-1}+1}^{k_{i}} a_{j} e_{j}\right)=\left\|\sum_{j=k_{i-1}+1}^{k_{i}} a_{j} e_{j}\right\|_{X}^{2} \text { and }\left\|u_{i}^{*}\right\|_{X^{*}} \leq C\left\|\sum_{j=k_{i-1}+1}^{k_{i}} a_{j} e_{j}\right\|_{X} .
$$

We now set

$$
y_{i}^{*}=\frac{u_{i}^{*}}{C\left(\sum_{i=1}^{n}\left\|\sum_{j=k_{i-1}+1}^{k_{i}} a_{j} e_{j}\right\|_{X}^{2}\right)^{1 / 2}} \text { for } 1 \leq i \leq n \text { and } y_{i}^{*}=0 \text { for } i>n .
$$


It is then clear that $y^{*}=\left(y_{i}^{*}\right)_{i=1}^{\infty} \in M$ and

$$
j\left(y^{*}\right)(x)=\frac{1}{C}\left(\left\|\sum_{j=k_{i-1}+1}^{k_{i}} a_{j} e_{j}\right\|_{X}^{2}\right)^{1 / 2} \geq \frac{\|x\|_{X^{\ell_{2}}}-\varepsilon}{C} .
$$

This finishes the proof of our claim.

Next we show that the Szlenk derivation on $K$ is controlled by the Szlenk derivation on $M$.

Claim 3.4. For any $\varepsilon>0$ and any countable ordinal $\xi$,

$$
s_{\varepsilon}^{\xi}(K) \subset j\left(s_{\varepsilon / 9}^{\xi}(M)\right) .
$$

Proof. We prove this by transfinite induction. The $\xi=0$ case is true since $K=j(M)$. If $\xi$ is a limit ordinal and $x^{*} \in s_{\varepsilon}^{\xi}(K)$, fix an increasing sequence $\left(\xi_{n}\right)_{n=1}^{\infty}$ tending to $\xi$ and note that, by the inductive hypothesis, there exists for each $n \in \mathbb{N}$ some $y_{n}^{*} \in s_{\varepsilon / 9}^{\xi_{n}}(M)$ such that $j y_{n}^{*}=x^{*}$. By passing to a subsequence, we may assume that $\left(y_{n}^{*}\right)_{n=1}^{\infty}$ is weak ${ }^{*}$-convergent to some $y^{*} \in \cap_{n=1}^{\infty} s_{\varepsilon / 9}^{\xi_{n}}(M)=s_{\varepsilon / 9}^{\xi}(M)$. Then using the weak*-continuity of $j$, we get that $j y^{*}=x^{*}$. This concludes the limit ordinal case.

For the successor case, assume $s_{\varepsilon}^{\xi}(K) \subset j\left(s_{\varepsilon / 9}^{\xi}(M)\right)$ and let $x^{*} \in s_{\varepsilon}^{\xi+1}(K)$. Fix a sequence $\left(x_{n}^{*}\right)_{n=1}^{\infty} \subset s_{\varepsilon}^{\xi}(K)$ converging weak ${ }^{*}$ to $x^{*}$ such that $\left\|x^{*}-x_{n}^{*}\right\|>\frac{\varepsilon}{3}$ for all $n \in \mathbb{N}$. For each $n \in \mathbb{N}$, we may pick $y_{n}^{*} \in s_{\varepsilon / 9}^{\xi}(M)$ such that $j y_{n}^{*}=x_{n}^{*}$. By passing to a subsequence, we may assume $\left(y_{n}^{*}\right)_{n=1}^{\infty}$ converges weak ${ }^{*}$ to some $y^{*} \in s_{\varepsilon / 9}^{\xi}(M)$ with $j y^{*}=x^{*}$. In order to see that $y^{*} \in s_{\varepsilon / 9}^{\xi+1}(M)$ and finish the claim, it is sufficient to see that

$$
\liminf _{n}\left\|y_{n}^{*}-y^{*}\right\| \geq \frac{\varepsilon}{9} .
$$

Seeking a contradiction, assume, after passing to a subsequence again, that

$$
\sup _{n \in \mathbb{N}}\left\|y_{n}^{*}-y^{*}\right\|<\frac{\varepsilon}{9} .
$$

Let $y_{n}^{*}=\left(y_{i, n}^{*}\right)_{i=1}^{\infty}$ and $y^{*}=\left(y_{i}^{*}\right)_{i=1}^{\infty}$. For each $n \in \mathbb{N}$, we may fix a sequence $\left(k_{i}^{n}\right)_{i=0}^{l_{n}}$ such that $l_{n} \in \mathbb{N} \cup\{\infty\}, 0=k_{0}^{n}, k_{i-1}^{n}<k_{i}^{n}$ for all $1 \leq i<l_{n}, y_{i, n}^{*} \in \operatorname{span}\left\{e_{j}^{*}: k_{i-1}^{n}<j \leq k_{i}^{n}\right\}$ for all $i<l_{n}$, and if $l_{n}<\infty, y_{l_{n}, n}^{*} \in \operatorname{span}\left\{e_{j}^{*}: j>k_{l_{n}-1}^{n}\right\}$ and $y_{i, n}^{*}=0$ for all $i>l_{n}$. By passing once more to a subsequence, we may assume that we are in one of the two following cases:

Case 1: $l_{n} \uparrow \infty$ and there exist $0=k_{0}<k_{1}<\ldots$ such that $k_{i}^{n} \underset{n}{\rightarrow} k_{i}$ for all $i \in \mathbb{N}$ and $I^{*} y_{i, n}^{*} \underset{n}{\rightarrow} I^{*} y_{i}^{*}$ in the $\left(X^{\ell_{2}}\right)^{*}$ norm for all $i \in \mathbb{N}$.

Case 2: There exists $l \in \mathbb{N}$ such that for each $0 \leq i<l, k_{i}^{n} \underset{n}{\rightarrow} k_{i}, k_{l}^{n} \underset{n}{\rightarrow} \infty, I^{*} y_{i, n}^{*} \underset{n}{\rightarrow} I^{*} y_{i}^{*}$ in the $\left(X^{\ell_{2}}\right)^{*}$ norm for all $i<l$, and $y_{i}^{*}=0$ for all $i>l$.

Let us consider the first case. Fix $N \in \mathbb{N}$ such that $\left(\sum_{i=N+1}^{\infty}\left\|y_{i}^{*}\right\|_{X^{*}}^{2}\right)^{1 / 2}<\frac{\varepsilon}{9}$ and note 
that, since $\left\|y_{n}^{*}-y^{*}\right\|<\frac{\varepsilon}{9},\left(\sum_{i=N+1}^{\infty}\left\|y_{i, n}^{*}\right\|_{X^{*}}^{2}\right)^{1 / 2}<\frac{2 \varepsilon}{9}$ for all $n \in \mathbb{N}$. Then

$$
\begin{aligned}
\frac{\varepsilon}{3} & \leq \liminf _{n}\left\|x_{n}^{*}-x^{*}\right\|_{\left(X^{\left.\ell_{2}\right)^{*}}\right.}=\liminf _{n}\left\|\sum_{i=1}^{\infty} I^{*} y_{i, n}^{*}-I^{*} y_{i}^{*}\right\|_{\left(X^{\ell_{2}}\right)^{*}} \\
& \leq \liminf _{n} \sum_{i=1}^{N}\left\|I^{*} y_{i, n}^{*}-I^{*} y_{i}^{*}\right\|_{\left(X^{\ell_{2}}\right)^{*}}+\left(\sum_{i=N+1}^{\infty}\left\|y_{i, n}^{*}\right\|_{X^{*}}^{2}\right)^{1 / 2}+\left(\sum_{i=N+1}^{\infty}\left\|y_{i}^{*}\right\|_{X^{*}}^{2}\right)^{1 / 2} \\
& <\frac{2 \varepsilon}{9}+\frac{\varepsilon}{9}=\frac{\varepsilon}{3} .
\end{aligned}
$$

This contradiction proves that the first case cannot occur. Now let us consider the second case. We similarly obtain the estimate

$$
\begin{aligned}
\frac{\varepsilon}{3} & \leq \liminf _{n}\left\|x_{n}^{*}-x^{*}\right\|_{\left(X^{\ell_{2}}\right)^{*}}=\liminf _{n}\left\|\sum_{i=1}^{\infty} I^{*} y_{i, n}^{*}-I^{*} y_{i}^{*}\right\|_{\left(X^{\left.\ell_{2}\right)^{*}}\right.} \\
& \leq \liminf _{n} \sum_{i=1}^{l-1}\left\|I^{*} y_{i, n}^{*}-I^{*} y_{i}^{*}\right\|_{\left(X^{\left.\ell_{2}\right)^{*}}\right.}+\left\|I^{*} y_{l, n}^{*}-I^{*} y_{l}^{*}\right\|_{\left(X^{\left.\ell_{2}\right)^{*}}\right.}+\left(\sum_{i=l+1}^{\infty}\left\|y_{i, n}^{*}\right\|_{X^{*}}^{2}\right)^{1 / 2} \\
& \leq\left\|y_{l, n}^{*}-y_{l}^{*}\right\|_{X^{*}}+\left(\sum_{i=l+1}^{\infty}\left\|y_{i, n}^{*}\right\|_{X^{*}}^{2}\right)^{1 / 2}<\frac{2 \varepsilon}{9}<\frac{\varepsilon}{3} .
\end{aligned}
$$

This contradiction concludes our proof.

Proof of Proposition 3.2. Now, as announced at the beginning of our proof, this last claim yields that $S z(K) \leq S z\left(\ell_{2}(X)\right)=S z(X)$. But our previous claim insured that $K \subset B_{\left(X^{\ell_{2}}\right)^{*}}$ is $c$-norming for $X^{\ell_{2}}$ and some $c>0$. It then follows from the geometric Hahn-Banach theorem that $c B_{\left(X^{\left.\ell_{2}\right)^{*}}\right.} \subset \overline{\mathrm{co}}^{*}(K)$. Finally we can apply Theorem 1.1 from [5] to deduce that $S z\left(X^{\ell_{2}}\right) \leq S z\left(\overline{\mathrm{co}}^{*}(K)\right) \leq S z(X)$. This finishes the proof of Proposition 3.2.

The construction of our family of spaces $\left(G_{\alpha}\right)_{\alpha \in \Gamma \backslash \Lambda}$ will also rely on the use of the Schreier families. So let us now recall the definition of the Schreier family $\mathcal{S}_{\alpha}$, for $\alpha$ a countable ordinal. We let $[\mathbb{N}]^{<\omega}$ denote the set of finite subsets of $\mathbb{N}$, which we identify with the set of empty or finite, strictly increasing sequences in $\mathbb{N}$. For each countable ordinal $\alpha, \mathcal{S}_{\alpha}$ will be a subset of $[\mathbb{N}]^{<\omega}$. For $E, F \in[\mathbb{N}]^{<\omega}$ and $n \in \mathbb{N}$, we write $E<F$ to mean $\max E<\min F$ and $n \leq E$ to mean $n \leq \min E$. We write $E \prec F$ to mean that $E$ is a proper initial segment of $F$, and $E \preceq F$ to mean that $E$ is an initial segment of $F$. We let

$$
\begin{gathered}
\mathcal{S}_{0}=\{\varnothing\} \cup\{(n): n \in \mathbb{N}\}, \\
\mathcal{S}_{\alpha+1}=\{\varnothing\} \cup\left\{\bigcup_{i=1}^{n} E_{i}: \varnothing \neq E_{i} \in \mathcal{S}_{\alpha}, E_{1}<\ldots<E_{n}, n \leq E_{1}\right\},
\end{gathered}
$$

and if $\alpha<\omega_{1}$ is a limit ordinal, we fix an increasing sequence $\left(\alpha_{n}\right)_{n=1}^{\infty}$ tending to $\alpha$ and let

$$
\mathcal{S}_{\alpha}=\left\{E \in[\mathbb{N}]^{<\omega}: \exists n \leq E \in \mathcal{S}_{\alpha_{n}}\right\}
$$


In what follows, $[\mathbb{N}]^{<\omega}$ will be topologized by the identification $[\mathbb{N}]^{<\omega} \ni E \leftrightarrow 1_{E} \in$ $\{0,1\}^{\mathbb{N}}$, where $\{0,1\}^{\mathbb{N}}$ has the Cantor topology.

Given $\left(m_{i}\right)_{i=1}^{k},\left(n_{i}\right)_{i=1}^{k}$ in $[\mathbb{N}]^{<\omega}$, we say $\left(n_{i}\right)_{i=1}^{k}$ is a spread of $\left(m_{i}\right)_{i=1}^{k}$ if $m_{i} \leq n_{i}$ for each $1 \leq i \leq k$.

We say a subset $\mathcal{F}$ of $[\mathbb{N}]^{<\omega}$ is

(i) spreading if it contains all spreads of its members,

(ii) hereditary if it contains all subsets of its members,

(iii) regular if it is spreading, hereditary, and compact.

Given $\mathcal{F}, \mathcal{G} \subset[\mathbb{N}]^{<\mathbb{N}}$, we let

$$
\mathcal{F}[\mathcal{G}]=\left\{\bigcup_{i=1}^{n} E_{i}: \varnothing \neq E_{i} \in \mathcal{G},\left(\min E_{i}\right)_{i=1}^{n} \in \mathcal{F}\right\} .
$$

We refer to [6] for a detailed presentation of these notions and their fundamentals properties.

We note that $\mathcal{F}[\mathcal{G}]$ is regular and if the Cantor-Bendixson indices of $\mathcal{F}$ and $\mathcal{G}$ are $\alpha+1$ and $\beta+1$, respectively, then the Cantor-Bendixson index of $\mathcal{F}[\mathcal{G}]$ is $\beta \alpha+1[6$, Proposition 3.1].

For each $n \in \mathbb{N}$, let

$$
\mathcal{A}_{n}=\left\{E \in[\mathbb{N}]^{<\omega}:|E| \leq n\right\} .
$$

It is well-known that for each $\alpha<\omega_{1}, \mathcal{S}_{\alpha}$ is regular with Cantor-Bendixson index $\omega^{\alpha}+1$. Moreover, for each $n \in \mathbb{N}, \mathcal{A}_{n}$ is regular with Cantor-Bendixson index $n+1$. These facts together with those cited from [6] yield the following.

Lemma 3.5. Fix an ordinal $\alpha<\omega_{1}$ and $n \in \mathbb{N}$.

(i) $\mathcal{A}_{n}\left[\mathcal{S}_{\alpha}\right]$ is regular with Cantor-Bendixson index $\omega^{\alpha} n+1$.

(ii) For any $\beta<\omega_{1}, \mathcal{S}_{\beta}\left[\mathcal{S}_{\alpha}\right]$ is regular with Cantor-Bendixson index $\omega^{\alpha+\beta}+1$.

Fact 3.6. If $\mathcal{F}$ and $\mathcal{G}$ are regular families, $E<F \neq \varnothing$, and $E, E \cup F \in \mathcal{F}[\mathcal{G}]$, then either $E \in \mathcal{F}^{\prime}[\mathcal{G}]$ or $F \in \mathcal{G}$.

Proof. Write $E \cup F=\cup_{i=1}^{n} E_{i}, \varnothing \neq E_{i} \in \mathcal{G}, E_{1}<\ldots<E_{n},\left(\min E_{i}\right)_{i=1}^{n} \in \mathcal{F}$.

If $E \cap E_{n}=\varnothing$, then there exists $1 \leq m \leq n$ such that $E \cap E_{i} \neq \varnothing$ for each $i<m$ and $E \cap E_{i}=\varnothing$ for each $m \leq i \leq n$.

If $m=1, E=\varnothing \in \mathcal{F}^{\prime}$, since $\varnothing \prec\left(\min E_{i}\right)_{i=1}^{n} \in \mathcal{F}$.

If $m>1$, the representation

$$
E=\bigcup_{i=1}^{m-1}\left(E \cap E_{i}\right)
$$

witnesses that $E \in \mathcal{F}^{\prime}[\mathcal{G}]$, since $\left(\min E_{i}\right)_{i=1}^{m-1} \in \mathcal{F}^{\prime}$.

Now if $E \cap E_{n} \neq \varnothing$, then $F=E_{n} \backslash E \subset E_{n}$, and $F \in \mathcal{G}$.

We are now ready to construct for each $\alpha \in \Gamma \backslash \Lambda$ a Banach space $G_{\alpha}$ such that $S z\left(G_{\alpha}\right)=\alpha$ and $S z\left(G_{\alpha}^{*}\right)=\omega$, while $G_{\alpha}$ is reflexive with an unconditional basis.

We write $\alpha=\omega^{\delta}$, where $0<\delta$. Then by standard facts about ordinals, either $\delta=\omega^{\xi}$ for some ordinal $\xi \in\left[0, \omega_{1}\right)$ or $\delta=\beta+\gamma$ for some $\beta, \gamma<\delta$. We shall separate our construction into these two main cases. 
So let us first suppose that $\delta=\omega^{\xi}$ with $\xi \in\left[0, \omega_{1}\right)$. Then $\xi$ must either be 0 or a successor ordinal, otherwise $\alpha \in \Lambda$.

If $\xi=0$, let $\mathcal{F}_{n}=\mathcal{S}_{0}$, for all $n \in \mathbb{N} \cup\{0\}$.

If $\xi=\zeta+1$, let $\mathcal{F}_{0}=\mathcal{S}_{0}$ and $\mathcal{F}_{n+1}=\mathcal{S}_{\omega \zeta}\left[\mathcal{F}_{n}\right]$ for $n \in \mathbb{N}$.

In both cases, denote

$$
K_{n}=\left\{2^{-n} \sum_{i \in E} e_{i}^{*}: E \in \mathcal{F}_{n}\right\} \text { for } n \in\{0\} \cup \mathbb{N} \text { and } K=\bigcup_{n=0}^{\infty} K_{n} .
$$

where $\left(e_{i}^{*}\right)_{i=1}^{\infty}$ is the the sequence of coordinate functionals defined on $c_{00}$, the space of finitely supported sequences.

Then we define $\mathfrak{G}_{\alpha}$ to be the completion of $c_{00}$ with respect to the norm

$$
\|x\|_{\mathfrak{G}_{\alpha}}=\sup _{x^{*} \in K}\left|x^{*}(x)\right| .
$$

Note that the canonical basis $\left(e_{i}\right)_{i=1}^{\infty}$ of $c_{00}$ is a 1-suppression unconditional basis of $\mathfrak{G}_{\alpha}$.

Finally, we set $G_{\alpha}=\mathfrak{G}_{\alpha}^{\ell_{2}}$, where this construction is meant with respect to the basis $\left(e_{i}\right)_{i=1}^{\infty}$, which we shall later call the canonical basis of $\mathfrak{G}_{\alpha}$.

It is easily checked that $\mathfrak{G}_{\omega}=c_{0}$ and $G_{\omega}=\ell_{2}$. So we clearly have that $G_{\omega}$ is reflexive with an unconditional basis and $S z\left(G_{\omega}\right)=S z\left(G_{\omega}^{*}\right)=\omega$. So we shall now assume that $\xi \neq 0$ and is therefore a countable successor ordinal.

Proposition 3.7. Assume $\alpha=\omega^{\omega^{\xi}}$, where $\xi$ is a countable successor ordinal. Then, $S z\left(\mathfrak{G}_{\alpha}\right) \leq \alpha$.

Proof. By [5, Theorem 1.1], it is sufficient to prove that $S z(K) \leq \alpha$, since $B_{\mathfrak{G}_{\alpha}^{*}}$ is the weak*-closed, absolutely convex hull of $K$.

First, it is easy to see that for any $\varepsilon>0$ and any ordinal $\eta$,

$$
s_{\varepsilon}^{\eta}(K) \subset\{0\} \cup \bigcup_{n=0} s_{\varepsilon}^{\eta}\left(K_{n}\right),
$$

whence

$$
S z(K, \varepsilon) \leq\left(\sup _{n \in \mathbb{N} \cup\{0\}} S z\left(K_{n}, \varepsilon\right)\right)+1 .
$$

Thus it suffices to show that $\sup _{n \in \mathbb{N} \cup\{0\}} S z\left(K_{n}, \varepsilon\right)<\alpha$ for each $\varepsilon>0$.

We note that the map $\phi_{n}: \mathcal{F}_{n} \rightarrow K_{n}$ given by $\phi_{n}(E)=\sum_{i \in E} e_{i}^{*}$ is a homeomorphism from $\mathcal{F}_{n}$ to $K_{n}$, where $K_{n}$ is endowed with its weak* topology. From this it follows that for any $n \in \mathbb{N} \cup\{0\}$ and any $\varepsilon>0$,

$$
S z\left(K_{n}, \varepsilon\right) \leq C B\left(K_{n}\right)=C B\left(\mathcal{F}_{n}\right) .
$$

We can write $\xi=\zeta+1$ with $\zeta \in\left[0, \omega_{1}\right)$. We now prove that if $2^{-m}<\varepsilon$, then for any $n>m$ and any ordinal $\eta$, then

$$
s_{2 \varepsilon}^{\eta}\left(K_{n}\right) \subset\left\{2^{-n} \sum_{i \in E} e_{i}^{*}: E \in \mathcal{F}_{m}^{\eta}\left[\mathcal{F}_{n-m}\right]\right\} .
$$


The proof is by induction on $\eta$, with the base case following from the fact that for any $a, b \in \mathbb{N}, \mathcal{F}_{a}\left[\mathcal{F}_{b}\right]=\mathcal{F}_{a+b}$. The limit ordinal case follows by taking intersections. Finally, assume we have the result for some $\eta$ and

$$
2^{-n} \sum_{i \in E} e_{i}^{*} \in s_{2 \varepsilon}^{\eta+1}\left(K_{n}, \varepsilon\right)
$$

so that the inductive hypothesis guarantees that $E \in \mathcal{F}_{m}^{\eta}\left[\mathcal{F}_{n-m}\right]$. Then there exists a sequence

$$
\left(2^{-n} \sum_{i \in E_{j}} e_{i}^{*}\right)_{j=1}^{\infty} \subset s_{2 \varepsilon}^{\eta}\left(K_{n}, \varepsilon\right) \subset\left\{2^{-n} \sum_{i \in E} e_{i}^{*}: E \in \mathcal{F}_{m}^{\eta}\left[\mathcal{F}_{n-m}\right]\right\}
$$

converging weak ${ }^{*}$ to $2^{-n} \sum_{j \in E} e_{i}^{*}$ and such that

$$
\left\|2^{-n} \sum_{i \in E} e_{i}^{*}-2^{-n} \sum_{i \in E_{j}} e_{i}^{*}\right\|>\varepsilon \text { for all } j \in \mathbb{N} .
$$

Of course, this means that $E_{j} \rightarrow E$ in $\mathcal{F}_{n}$ so that, after passing to another subsequence, we may assume $E_{j}=E \cup F_{j}$ for some $F_{j} \neq \varnothing$ with $E<F_{j}$. Now since $E, E_{j} \in$ $\mathcal{F}_{m}^{\eta}\left[\mathcal{F}_{n-m}\right]$ for each $j$, by Fact 3.6, either $F_{j} \in \mathcal{F}_{n-m}$ or $E \in \mathcal{F}_{m}^{\eta+1}\left[\mathcal{F}_{n-m}\right]$. However, if $F_{j} \in \mathcal{F}_{n-m}$, then $2^{m-n} \sum_{i \in F_{j}} e_{i}^{*} \in B_{\mathfrak{G}_{\alpha}^{*}}$ and

$$
\left\|2^{-n} \sum_{i \in E} e_{i}^{*}-2^{-n} \sum_{i \in E_{j}} e_{i}^{*}\right\|=2^{-m}\left\|2^{m-n} \sum_{i \in F_{j}} e_{i}^{*}\right\| \leq 2^{-m}<\varepsilon,
$$

a contradiction. This concludes the successor case.

We now deduce from the inclusion we just proved, that

$$
s_{2 \varepsilon}^{\omega^{\omega \zeta_{m}}+1}\left(K_{n}\right) \subset\left\{2^{-n} \sum_{i \in E} e_{i}^{*}: E \in \mathcal{F}_{m}^{\omega^{\omega^{\zeta}} m}+1\left[\mathcal{F}_{n-m}\right]\right\}=\varnothing .
$$

So, we can now estimate

$$
S z\left(K_{n}, 2 \varepsilon\right) \leq \begin{cases}\omega^{\omega^{\zeta} n}+1 & : n \leq \log _{2}(1 / \varepsilon) \\ \omega^{\omega^{\zeta}\left\lceil\log _{2}(1 / \varepsilon)\right\rceil}+1 & : n>\log _{2}(1 / \varepsilon),\end{cases}
$$

and this estimate finishes the proof of our proposition.

Corollary 3.8. Assume $\alpha=\omega^{\omega^{\xi}}$, where $\xi$ is a countable successor ordinal. Then $G_{\alpha}$ is reflexive with an unconditional basis, $S z\left(G_{\alpha}\right)=\alpha$, and $S z\left(G_{\alpha}^{*}\right)=\omega$.

Proof. Since the canonical basis of $\mathfrak{G}_{\alpha}$ is 1-suppression unconditional, it is clearly a 1suppression unconditional basis for $G_{\alpha}$. Our previous corollary insures that $S z\left(\mathfrak{G}_{\alpha}\right) \leq \alpha$ and therefore that $\mathfrak{G}_{\alpha}$ does not contain $\ell_{1}$. It then follows from a classical result of R.C. James [10] that the canonical basis of $\mathfrak{G}_{\alpha}$ is shrinking. Thus we can apply Proposition 3.1 that $G_{\alpha}$ is reflexive and $S z\left(G_{\alpha}^{*}\right)=\omega$.

We also deduce from Proposition 3.2 that $S z\left(G_{\alpha}\right) \leq S z\left(\mathfrak{G}_{\alpha}\right)=\alpha$. 
We now have to prove that $S z\left(G_{\alpha}\right) \geq \alpha$. So let us write again $\alpha=\omega^{\omega^{\zeta+1}}$, with $\zeta \in\left[0, \omega_{1}\right)$. Suppose $n \in \mathbb{N}$ and $E<F$ are such that $F \in \mathcal{F}_{n}$. Fix $k \in F \backslash E$. Note that

$$
2^{-n} \sum_{i \in F} e_{i}^{*} \in K_{n}
$$

and

$$
\left\|2^{-n} \sum_{i \in E} e_{i}^{*}-2^{-n} \sum_{i \in F} e_{i}^{*}\right\|_{G_{\alpha}} \geq\left|\left(2^{-n} \sum_{i \in E} e_{i}^{*}-2^{-n} \sum_{i \in F} e_{i}^{*}\right)\left(e_{k}\right)\right|=2^{-n},
$$

since $\left\|e_{k}\right\|_{G_{\alpha}}=1$. From this and an easy induction argument, we see that for any $n \in \mathbb{N}$, any $0 \leq \mu<C B\left(\mathcal{F}_{n}\right)$ and any $E \in \mathcal{F}_{n}^{\mu}, 2^{-n} \sum_{i \in E} e_{i}^{*} \in s_{2^{-n-1}}^{\mu}\left(B_{G_{\alpha}^{*}}\right)$. Since $C B\left(\mathcal{F}_{n}\right)=\left(\omega^{\omega^{\zeta}}\right)^{n}=\omega^{\omega^{\zeta} n}$, we deduce that

$$
S z\left(G_{\alpha}\right) \geq \sup _{n \in \mathbb{N}} \omega^{\omega^{\zeta} n}=\omega^{\omega^{\zeta+1}}=\alpha .
$$

This finishes the proof and our construction for $\alpha=\omega^{\omega^{\xi}}$, with $\xi$ being a countable successor ordinal.

We will now modify slightly our construction in order to treat the case in which $\alpha=\omega^{\beta+\gamma}$, with $\omega^{\beta}<\alpha$ and $\omega^{\gamma}<\alpha$. We have to consider two subcases.

First suppose $\gamma$ is a limit ordinal. We fix $\gamma_{0}=0$ and an increasing sequence $\left(\gamma_{n}\right)_{n=1}^{\infty}$ such that $\sup _{n \in \mathbb{N}} \gamma_{n}=\gamma$. Then we set

$$
\mathcal{F}_{0}=\mathcal{S}_{\beta} \text { and } \mathcal{F}_{n}=\mathcal{S}_{\gamma_{n}}\left[\mathcal{S}_{\beta}\right], \text { for } n \in \mathbb{N}
$$

If $\gamma=\zeta+1$ is a successor ordinal, we set

$$
\mathcal{F}_{0}=\mathcal{S}_{\beta+\zeta} \text { and } \mathcal{F}_{n}=\mathcal{A}_{n}\left[\mathcal{S}_{\beta+\zeta}\right], \text { for } n \in \mathbb{N} .
$$

In either case, let

$$
K_{n}=\left\{2^{-n} \sum_{i \in E} e_{i}^{*}: E \in \mathcal{F}_{n}\right\} \text { for } n \in\{0\} \cup \mathbb{N} \text { and } K=\bigcup_{n=0}^{\infty} K_{n} .
$$

As in our first situation, we define $\mathfrak{G}_{\alpha}$ to be the completion of $c_{00}$ with respect to the norm $\|x\|_{\mathfrak{G}_{\alpha}}=\sup _{x^{*} \in K}\left|x^{*}(x)\right|$ and let $G_{\alpha}=\mathfrak{G}_{\alpha}^{\ell_{2}}$, where this construction is meant with respect to the canonical basis of $\mathfrak{G}_{\alpha}$.

Proposition 3.9. Assume that $\alpha$ is a countable ordinal that can be written $\alpha=\omega^{\beta+\gamma}$, with $\omega^{\beta}<\alpha$ and $\omega^{\gamma}<\alpha$. Then $S z\left(\mathfrak{G}_{\alpha}\right) \leq \alpha$.

Proof. Again, it is sufficient to show that $S z(K) \leq \alpha$. Arguing as in Proposition 3.7, we first note that for any $\varepsilon>0$ and $n \in \mathbb{N}$,

$$
S z\left(K_{n}, \varepsilon\right) \leq C B\left(\mathcal{F}_{n}\right)= \begin{cases}\omega^{\beta+\gamma_{n}}+1 & : \gamma \text { a limit } \\ \omega^{\beta+\mu} n+1 & : \gamma=\zeta+1 .\end{cases}
$$

Now for $n \in \mathbb{N}$ and $\varepsilon>0$ such that $2^{-n}<\varepsilon$, we claim that for any ordinal $\eta$,

$$
s_{2 \varepsilon}^{\eta}\left(K_{n}\right) \subset \begin{cases}\left\{2^{-n} \sum_{i \in E} e_{i}^{*}: E \in \mathcal{S}_{\gamma_{n}}^{\eta}\left[\mathcal{S}_{\beta}\right]\right\} & : \gamma \text { a limit } \\ \left.2^{-n} \sum_{i \in E} e_{i}^{*}: E \in \mathcal{A}_{n}^{\eta}\left[\mathcal{S}_{\beta+\zeta}\right]\right\} & : \gamma=\zeta+1 .\end{cases}
$$


The proof is even easier than the analogous claim in the proof of Proposition 3.8, so we omit it. Note that in particular, when $\gamma$ is a limit ordinal and $2^{-n}<\varepsilon, \mathcal{S}_{\gamma_{n}}^{\omega^{\gamma}}=\varnothing$, whence the previous claim yields the estimate $S z\left(K_{n}, 2 \varepsilon\right) \leq \omega^{\gamma}<\omega^{\beta+\gamma}$ when $2^{-n}<\varepsilon$. Similarly, since $\mathcal{A}_{n}^{\omega}=\varnothing, S z\left(K_{n}, 2 \varepsilon\right) \leq \omega<\omega^{\beta+\zeta+1}$ when $2^{-n}<\varepsilon$.

Therefore for $n \leq \log _{2}(1 / \varepsilon)$,

$$
S z\left(K_{n}, 2 \varepsilon\right) \leq C B\left(\mathcal{F}_{n}\right)= \begin{cases}\omega^{\beta+\gamma_{n}}+1 & : \gamma \text { a limit } \\ \omega^{\beta+\mu} n+1 & : \gamma=\zeta+1\end{cases}
$$

and for $n>\log _{2}(1 / \varepsilon)$,

$$
S z\left(K_{n}, 2 \varepsilon\right) \leq \begin{cases}\omega^{\gamma} & : \gamma \text { a limit } \\ \omega & : \gamma=\zeta+1\end{cases}
$$

Thus in either case, for every $\varepsilon>0, \sup _{n \in \mathbb{N} \cup\{0\}} S z\left(K_{n}, \varepsilon\right)<\alpha$, yielding the result.

Corollary 3.10. Assume that $\alpha$ is a countable ordinal that can be written $\alpha=\omega^{\beta+\gamma}$, with $\omega^{\beta}<\alpha$ and $\omega^{\gamma}<\alpha$. Then $G_{\alpha}$ is reflexive with an unconditional basis, $S z\left(G_{\alpha}\right)=$ $\alpha$, and $S z\left(G_{\alpha}^{*}\right)=\omega$.

Proof. The proof is the same as the proof of Corollary 3.8, only noting that $C B\left(\mathcal{F}_{n}\right)=$ $\omega^{\beta+\gamma_{n}}+1$ when $\gamma$ is a limit ordinal and $C B\left(\mathcal{F}_{n}\right)=\omega^{\beta+\zeta} n+1$ if $\gamma=\zeta+1$.

\subsection{Proof of Theorem 1.1.}

We now conclude with the proof of Theorem 1.1.

Proof. For the proof we fix $\alpha \in \Gamma \backslash \Lambda$ and do an induction on $n \in \mathbb{N}$.

For $n=1$, the result is given by Theorem 1.3.

For $n=2$, let $X_{\alpha}$ (given by Theorem 1.5 in [5]) be a separable Banach space such that $S z\left(X_{\alpha}\right)=\alpha$. Then denote $Z$ the Lindenstrauss space such that $Z^{* *} / Z$ is isomorphic to $X_{\alpha}$. We have, by Theorem 1.2 that $S z(Z)=S z\left(Z^{*}\right)=\omega$. Next, using Proposition 2.1 in [4] we get that there exists $C>0$ such that

$$
\forall \varepsilon>0 \quad S z\left(Z^{* *}, \varepsilon\right) \leq S z\left(Z^{* *} / Z, \frac{\varepsilon}{C}\right) S z\left(Z, \frac{\varepsilon}{C}\right)<\alpha .
$$

The last inequality follows from the fact that $S z\left(Z^{* *} / Z, \frac{\varepsilon}{C}\right)<\alpha, S z(Z, \varepsilon)<\omega$ and elementary properties of the multiplication of ordinal numbers. We deduce that $S z\left(Z^{* *}\right)$ is at most $\alpha$ and therefore $S z\left(Z^{* *}\right)=\alpha$, since $S z\left(Z^{* *}\right) \geq S z\left(Z^{* *} / Z\right)=S z\left(X_{\alpha}\right)=\alpha$.

Assume now that $n \geq 3$ and that our statement has been proved for $1 \leq k \leq n-1$. By our induction hypothesis there exists a separable Banach space $X$ such that $S z(X)=$ $. .=S z\left(X^{(n-3)}\right)=\omega$ and $S z\left(X^{(n-2)}\right)=\alpha$. Then denote $Z$ the Lindenstrauss space such that $Z^{* *} / Z$ is isomorphic to $X$. We already know that $S z(Z)=S z\left(Z^{*}\right)=\omega$. Since $S z(X)=\omega$, we can use the fact that having a Szlenk index equal to $\omega$ is a three space property (see [4]) to deduce that $S z\left(Z^{* *}\right)=\omega$. Then using elementary facts about duality, we have that for all $k \geq 3$ the space $Z^{(k)}$ is isomorphic to $Z^{(k-2)} \oplus X^{(k-2)}$ which implies that $S z\left(Z^{(k)}\right)=\max \left\{S z\left(Z^{(k-2)}\right), S z\left(X^{(k-2)}\right)\right\}$ (see [6]). It now clearly follows that $S z\left(Z^{(k)}\right)=\omega$ for all $k \in\{0, . ., n-1\}$ and $S z\left(Z^{(n)}\right)=\alpha$. 
Acknowledgements. This work was initiated while the second named author was visiting Miami University in Oxford, Ohio. He wishes to thank the Mathematics Department of Miami University for this invitation and its hospitality.

\section{REFERENCES}

[1] D. Alspach, R. Judd, and E. Odell, The Szlenk index and local $\ell_{1}$-indices, Positivity, 9 (2005), no. $1,1-44$.

[2] C. Bessaga and A. Pełczyński A., Spaces of continuous functions (IV) (on isomorphical classification of spaces of continuous functions), Studia Math., 19 (1960), 53-62.

[3] P.A.H. Brooker, Direct sums and the Szlenk index. J. Funct. Anal. 260 (2011), no. 8, 2222-2246.

[4] P.A.H. Brooker and G. Lancien, Three-space property for asymptotically uniformly smooth renormings, Journal of Math. Anal. and Appl. 398 (2013), 867-871.

[5] R.M Causey, The Szlenk index of injective tensor products and convex hulls, J. Funct. Anal. 272 (2017), no. 8, 3375-3409.

[6] R.M Causey, Concerning the Szlenk index. Studia Math. 236 (2017), no. 3, 201-244.

[7] R.M Causey, Power type asymptotically uniformly smooth norms and asymptotically uniformly flat norms. Preprint. arXiv:1705.05484v1

[8] S. Dilworth, D. Kutzarova, G. Lancien and L. Randrianarivony, Equivalent norms with the property $\beta$ of Rolewicz, Revista de la Real Academia de Ciencias Exactas, Físicas y Naturales. Serie A. Matemáticas, 111 (2017), no. 1, 101-113.

[9] G. Godefroy, N. J. Kalton and G. Lancien, Szlenk indices and uniform homeomorphisms, Trans. Amer. Math. Soc., 353 (2001), 3895-3918.

[10] , R.C. James, Bases and reflexivity of Banach spaces, Annals of Math., 52 (1950), 518-527.

[11] W.B. Johnson, J. Lindenstrauss, D. Preiss and G. Schechtman, Almost Fréchet differentiability of Lipschitz mappings between infinite-dimensional Banach spaces, Proc. London Math. Soc., 84 (2002), no. 3, 711-746.

[12] H. Knaust, E. Odell and T. Schlumprecht, On asymptotic structure, the Szlenk index and UKK properties in Banach spaces, Positivity, 3 (1999), 173-199.

[13] G. Lancien, On the Szlenk index and the weak* dentability index, Quarterly J. Math. Oxford, 47 (1996), 59-71.

[14] On James' paper "Separable conjugate spaces", Israel J. Math 9 (1971), 279-284.

[15] J. Lindenstrauss and L. Tzafriri, Classical Banach spaces, Vol.1, Sequence spaces, Springer, Berlin 1977.

[16] V.D. Milman, Geometric theory of Banach spaces. II. Geometry of the unit ball (Russian), Uspehi Mat. Nauk, 26, (1971), 73-149. English translation: Russian Math. Surveys 26, (1971), 79-163.

[17] , E. Odell, Th. Schlumprecht, A. Zsák, A new infinite game in Banach spaces with applications. Banach spaces and their applications in analysis, 147?82, Walter de Gruyter, Berlin, 2007.

[18] C. Samuel, Indice de Szlenk des $C(K)$, Publications Mathématiques de l'Université Paris VII, Séminaire de Géométrie des espaces de Banach, Vol. I-II, Paris (1983), 81-91.

[19] W. Szlenk, The non existence of a separable reflexive space universal for all separable reflexive Banach spaces, Studia Math., 30 (1968), 53-61.

R.M. Causey, Department of Mathematics, Department of Mathematics, Miami UniverSITY, OXFORD, OH 45056, USA

E-mail address: causeyrm@miamioh.edu

Laboratoire de Mathématiques de Besançon, Université Bourgogne Franche-Comté, CNRS UMR-6623, 16 route de Gray, 25030 Besançon Cédex, Besançon, France

E-mail address: gilles.lancien@univ-fcomte.fr 